\title{
Qualitative Behaviour of Elastic-Plastic Solutions for a Class of Damage Mechanics Models Near Bimaterial Interfaces: A Simple Analytical Example
}

\author{
Sergei Alexandrov ${ }^{1}$ and Yusof Mustafa $^{2}$ \\ ${ }^{1}$ A.Yu. Ishlinskii Institute for Problems in Mechanics, Russian Academy of Sciences, 101-1 Prospect Vernadskogo, \\ Moscow 119526, Russia \\ ${ }^{2}$ Faculty of Mechanical Engineering, Universiti Teknologi Malaysia, 81310 Skudai, Johor, Malaysia \\ Correspondence should be addressed to Sergei Alexandrov; sergei_alexandrov@spartak.ru
}

Received 8 August 2013; Revised 22 November 2013; Accepted 24 November 2013

Academic Editor: Yuri Petryna

Copyright (c) 2013 S. Alexandrov and Y. Mustafa. This is an open access article distributed under the Creative Commons Attribution License, which permits unrestricted use, distribution, and reproduction in any medium, provided the original work is properly cited.

\begin{abstract}
The paper presents an exact analytic solution for a class of elastic-plastic models with damage evolution. The boundary value problem consists of a planar deformation comprising the simultaneous shearing and expansion of a hollow cylindrical specimen of material and involves a bimaterial interface at which the materials stick to each other. With no loss of generality for understanding the qualitative behaviour of the solution near the bimaterial interface, an extreme case when the hard material is rigid is considered. The solution is reduced to a transcendental equation for the value of the equivalent plastic strain at the bimaterial interface. This equation predicts that the equivalent plastic strain attains a maximum under certain conditions. The existence of the solution of the boundary value problem depends on the value of the damage parameter at fracture, which is a material constant. In particular, if this value is larger than the value of the damage parameter at the bimaterial interface corresponding to the maximum possible value of the equivalent strain at this interface, then no solution exists. Experimental data available in the literature are used to assess whether Lemaitre's model is applicable.
\end{abstract}

\section{Introduction}

The ductile damage evolution equations of continuum mechanics can be conveniently divided into three groups: (i) uncoupled models, (ii) partly coupled models, and (iii) coupled models. In the case of the models of group (i) the damage evolution equation is solved having found the solution for the system of equations of plasticity theory. A recent review of models of this group is provided in [1]. The models of group (iii) include the fully coupled system of equations of plasticity theory and the damage evolution equation. In particular, the material is plastically compressible. A recent review of models of this group is provided in [2]. In the present paper, a wide class of models of group (ii) for elastic-plastic materials is adopted. These models containing one scalar measure of damage are based on the pressure-independent yield criterion and the associated flow rule for plastic strain rates. The evolution of the damage parameter is controlled by an additional equation. Such models are presented, for example, in [3-7]. The yield criterion and elastic moduli depend on the damage parameter. Many structures and machine parts are composed of two or more different materials and, therefore, contain bimaterial interfaces. Solution behaviour in the vicinity of such interfaces may reveal some qualitative features that provide an assessment of the model adopted to find the solution. For example, using a rigid perfectly plastic material model, it has been shown in [8] that the velocity field is singular in the vicinity of some bimaterial interfaces. This singularity in the velocity field is not compatible with the class of damage evolution models reviewed in [9]. In order to assess the mathematical model, it is possible to study any boundary value problem, even if its practical application is not feasible. It is more important to find the exact solution of the problem without relaxing the boundary conditions and field equations. Such a boundary value problem has been proposed and solved for a rigid 
plastic linear hardening material model in [10]. Then, this boundary value problem has been successfully used in $[11,12]$ to study various qualitative features of solutions for several material models. However, all of these solutions are for rigid plastic materials with no damage evolution. In the present paper, the same boundary value problem is combined with a class of elastic-plastic models with damage evolution at small strains. The solution is reduced to a transcendental equation for the value of the equivalent plastic strain at the bimaterial interface. The solution to this equation predicts that this value attains a maximum under certain conditions. The existence of the solution of the boundary value problem depends on the value of the damage parameter at fracture, which is supposed to be a constitutive parameter. In particular, if the value of the damage parameter at the bimaterial interface corresponding to the maximum possible value of the equivalent plastic strain at this interface is smaller than the damage parameter at fracture, then no solution exists. The model proposed by Lemaitre [3] is studied in some detail. In particular, experimental data provided in [13] are used to assess whether the model for this specific set of constitutive parameters is applicable (in the sense that the solution exists).

\section{Statement of the Problem}

Consider an infinite elastic-plastic circular cylinder of internal radius $r_{0}$ and external radius $R_{0}$ subject to the action of tangential stresses uniformly distributed over its external radius. It is assumed that a rigid rod of radius $r_{0}$ is inserted into the hole of the cylinder. The rod is motionless and fixed to the cylinder. Due to this system of loading and constraints, the cylinder is twisted. It is convenient to introduce a cylindrical polar coordinate system $(r, \theta, z)$ with its $z$-axis coinciding with the axis of symmetry of the cylinder. The boundary conditions are

$$
u_{\theta}=0
$$

for $r=r_{0}$ and

$$
u_{\theta}=\omega R_{0}
$$

for $r=R_{0}$. Here $u_{\theta}$ is the circumferential displacement and $\omega$ is the angular displacement of the external surface of the cylinder.

A typical dependence of the shear yield stress, $k$, on the equivalent plastic strain, $\varepsilon_{\mathrm{eq}}^{p}$, and damage parameter, $D$, is $[3$, $4,7]$

$$
k=k_{0}\left[1+f\left(\varepsilon_{\mathrm{eq}}^{p}\right)\right](1-D)
$$

Here $k_{0}$ is the initial shear yield stress when $\varepsilon_{\mathrm{eq}}^{p}=0$ and $D=0$. The function $f\left(\varepsilon_{\mathrm{eq}}^{p}\right)$ satisfies the conditions $f(0)=0$ and $d f / d \varepsilon_{\mathrm{eq}}^{p}>0$ for any $\varepsilon_{\mathrm{eq}}^{p}$. The equivalent plastic strain is defined by the equation

$$
\frac{d \varepsilon_{\mathrm{eq}}^{p}}{d t}=\xi_{\mathrm{eq}}^{p} .
$$

Here $t$ is the time and $d / d t$ is the convected derivative. The equivalent plastic strain rate, $\xi_{\text {eq }}^{p}$, is defined by

$$
\xi_{\mathrm{eq}}^{p}=\sqrt{\frac{2}{3} \xi_{i j}^{p} \xi_{i j}^{p}}
$$

where $\xi_{i j}^{p}$ are the plastic strain rate components. The yield criterion is

$$
\tau_{i j} \tau_{i j}=2 k^{2}
$$

where $\tau_{i j}$ are the deviatoric components of the stress tensor. The flow rule associated with (6) is

$$
\xi_{i j}^{p}=\lambda \tau_{i j}
$$

where $\lambda$ is a scalar factor of proportionality. The elastic strains, $\varepsilon_{i j}^{e}$, obey Hooke's law. The total strains are

$$
\varepsilon_{i j}=\varepsilon_{i j}^{e}+\varepsilon_{i j}^{p}
$$

In the case of isotropic models with a single scalar measure of damage typical damage evolution equations are $[3,4,7]$

$$
\begin{gathered}
\frac{d D}{d t}=F\left(\frac{\sigma}{\sqrt{3} k}, D\right) \xi_{\mathrm{eq}}^{p} \quad \text { if } \varepsilon_{\mathrm{eq}}^{p} \geq \varepsilon_{0}, \\
\frac{d D}{d t}=0 \quad \text { if } \varepsilon_{\mathrm{eq}}^{p}<\varepsilon_{0}, \\
\frac{d D}{d t}=F\left(\frac{\sigma}{\sqrt{3} k}, D\right)\left(\varepsilon_{\mathrm{eq}}^{p}\right)^{1 / M} \xi_{\mathrm{eq}}^{p} \text { if } \varepsilon_{\mathrm{eq}}^{p} \geq \varepsilon_{0}, \\
\frac{d D}{d t}=0 \quad \text { if } \varepsilon_{\mathrm{eq}}^{p}<\varepsilon_{0} .
\end{gathered}
$$

Here $\sigma$ is the hydrostatic stress, $\varepsilon_{0}$ is the threshold equivalent plastic strain for the initiation of damage, $M$ is constant, and $F$ is a given function of its arguments. The fracture criterion is

$$
D=D_{c}
$$

where $D_{c}$ is a material constant whose value for metallic materials lies in the range $0.2<D_{c}<0.8$ [7]. Equations (3) to (11) along with Hooke's law represent a typical isotropic elastic-plastic material model with damage evolution at small strains. These equations should be supplemented with the equilibrium equations.

\section{Solution}

In this section, an exact solution of the equations of the previous section is derived in the cylindrical coordinates assuming that the solution is independent of $\theta$ and $z$. It is then demonstrated that the solution is compatible with the boundary conditions (1) and (2). Suppose that all components of the stress tensor vanish except for the shear component $\tau_{r \theta}$. The yield criterion (6) is satisfied if

$$
\tau_{r \theta}=k .
$$


The only nontrivial equilibrium equation is

$$
\frac{\partial \tau_{r \theta}}{\partial r}+\frac{2 \tau_{r \theta}}{r}=0
$$

Denote the radius of the elastic-plastic boundary by $R, r_{0} \leq$ $R \leq R_{0}$. The material just on the elastic side of the boundary must be on the point of yielding. Therefore, $\tau_{r \theta}=k=k_{0}$ at $r=R$. Integrating (13) with the use of this condition gives

$$
\frac{\tau_{r \theta}}{k_{0}}=\left(\frac{R}{r}\right)^{2}
$$

It follows from Hooke's law that

$$
2 G \varepsilon_{r \theta}^{e}=\tau_{r \theta},
$$

where $G$ is the modulus of shear.

Consider the elastic region, $R \leq r \leq R_{0}$. In this region, $\varepsilon_{i j}^{e}=\varepsilon_{i j}$ and $G=G_{0}$, where $G_{0}$ is the shear modulus of undamaged material. Therefore, substituting (14) into (15) yields

$$
\varepsilon_{r \theta}=\frac{k_{0}}{2 G_{0}}\left(\frac{R}{r}\right)^{2} .
$$
gives

In the plastic region, $r_{0} \leq r \leq R$, combining (12) and (14)

$$
\frac{k}{k_{0}}=\left(\frac{R}{r}\right)^{2} .
$$

A typical assumption is that $G=G_{0}(1-D)[3,4,7]$. Then, substituting (12) into (15) results in

$$
\varepsilon_{r \theta}^{e}=\frac{k}{2 G_{0}(1-D)} .
$$

Since the only nonzero stress component is $\tau_{r \theta}$, it follows from (7) that the only nonzero plastic strain rate component is $\xi_{r \theta}^{p}$. Then, (5) reduces to

$$
\xi_{\mathrm{eq}}^{p}=\frac{2}{\sqrt{3}} \xi_{r \theta}^{p}
$$

It has been taken into account here that $\tau_{r \theta}>0$, as follows from (12). Since strains are small, $\xi_{\mathrm{eq}}^{p}=\partial \varepsilon_{\mathrm{eq}}^{p} / \partial t$ and $\xi_{r \theta}^{p}=$ $\partial \varepsilon_{r \theta}^{p} / \partial t$. Therefore, integrating (19) with respect to $t$ gives

$$
\varepsilon_{\mathrm{eq}}^{p}=\frac{2}{\sqrt{3}} \varepsilon_{r \theta}^{p}
$$

The total shear strain in the plastic region is found from (8), (18), and (20) as

$$
\varepsilon_{r \theta}=\frac{k}{2 G_{0}(1-D)}+\frac{\sqrt{3}}{2} \varepsilon_{\mathrm{eq}}^{p} .
$$

Since $\sigma=0$, (9) becomes

$$
\begin{gathered}
\frac{d D}{d t}=F_{1}(D) \xi_{\mathrm{eq}}^{p} \quad \text { if } \varepsilon_{\mathrm{eq}}^{p} \geq \varepsilon_{0}, \\
\frac{d D}{d t}=0 \quad \text { if } \varepsilon_{\mathrm{eq}}^{p}<\varepsilon_{0},
\end{gathered}
$$

where $F_{1}(D)=F(0, D)$. Assuming that $D=0$ at the initial instant, (22) can be integrated to give

$$
\begin{gathered}
\Omega(D)=\varepsilon_{\mathrm{eq}}^{p}-\varepsilon_{0} \quad \text { if } \varepsilon_{\mathrm{eq}}^{p} \geq \varepsilon_{0} \\
D=0 \quad \text { if } \varepsilon_{\mathrm{eq}}^{p}<\varepsilon_{0} .
\end{gathered}
$$

Here

$$
\Omega(D)=\int_{0}^{D} \frac{d x}{F_{1}(x)}
$$

where $x$ is a dummy variable of integration. It follows from (3), (17), and (23) that

$$
\left(\frac{R}{r}\right)^{2}=\left[1+f\left(\varepsilon_{\mathrm{eq}}^{p}\right)\right]\left[1-\Omega^{-1}\left(\varepsilon_{\mathrm{eq}}^{p}-\varepsilon_{0}\right)\right],
$$

where $\Omega^{-1}$ is the function inverse to $\Omega$. It has been assumed here that $\varepsilon_{\mathrm{eq}}^{p} \geq \varepsilon_{0}$. Substituting (3) into (21) yields

$$
\varepsilon_{r \theta}=\frac{k_{0}\left[1+f\left(\varepsilon_{\mathrm{eq}}^{p}\right)\right]}{2 G_{0}}+\frac{\sqrt{3}}{2} \varepsilon_{\mathrm{eq}}^{p} .
$$

For the problem under consideration, the total shear strain is defined by

$$
\varepsilon_{r \theta}=\frac{1}{2}\left(\frac{\partial u_{\theta}}{\partial r}-\frac{u_{\theta}}{r}\right)=\frac{r}{2} \frac{\partial\left(u_{\theta} / r\right)}{\partial r} .
$$

Combining (26) and (27) gives

$$
r \frac{\partial\left(u_{\theta} / r\right)}{\partial r}=\frac{k_{0}\left[1+f\left(\varepsilon_{\mathrm{eq}}^{p}\right)\right]}{G_{0}}+\sqrt{3} \varepsilon_{\mathrm{eq}}^{p} .
$$

It is convenient to introduce new independent variables as

$$
\rho=\frac{R}{r}, \quad s=\frac{R}{R_{0}} .
$$

Then,

$$
\frac{\partial}{\partial r}=-\frac{\rho^{2}}{R} \frac{\partial}{\partial \rho}, \quad \frac{\partial}{\partial t}=\frac{\dot{R}}{R}\left(\rho \frac{\partial}{\partial \rho}+s \frac{\partial}{\partial s}\right),
$$

where $\dot{R} \equiv d R / d t$. Using (29), (30), (25) and (28) transforms to

$$
\begin{aligned}
& \rho^{2}=\left[1+f\left(\varepsilon_{\mathrm{eq}}^{p}\right)\right]\left[1-\Omega^{-1}\left(\varepsilon_{\mathrm{eq}}^{p}-\varepsilon_{0}\right)\right] \equiv \Lambda\left(\varepsilon_{\mathrm{eq}}^{p}\right), \\
& -\frac{\rho \partial\left(u_{\theta} / r\right)}{\partial \rho}=\frac{k_{0}\left[1+f\left(\varepsilon_{\mathrm{eq}}^{p}\right)\right]}{G_{0}}+\sqrt{3} \varepsilon_{\mathrm{eq}}^{p} \equiv \Phi\left(\varepsilon_{\mathrm{eq}}^{p}\right),
\end{aligned}
$$

respectively. Eliminating $\rho$ between (31) and (32) leads to

$$
\frac{\partial\left(u_{\theta} / r\right)}{\partial \varepsilon_{\mathrm{eq}}^{p}}=-\frac{\Phi\left(\varepsilon_{\mathrm{eq}}^{p}\right) \Lambda^{\prime}\left(\varepsilon_{\mathrm{eq}}^{p}\right)}{2 \Lambda\left(\varepsilon_{\mathrm{eq}}^{p}\right)},
$$


where $\Lambda^{\prime}\left(\varepsilon_{\mathrm{eq}}^{p}\right) \equiv d \Lambda\left(\varepsilon_{\mathrm{eq}}^{p}\right) / d \varepsilon_{\mathrm{eq}}^{p}$. Let $u_{R}$ be the value of $u_{\theta}$ at $r=R$. Since $\varepsilon_{\mathrm{eq}}^{p}=0$ at $r=R$, the solution to (33) is

$$
\frac{u_{\theta}}{r}=-\frac{1}{2} \int_{0}^{\varepsilon_{\mathrm{eq}}^{p}} \frac{\Phi(x) \Lambda^{\prime}(x)}{\Lambda(x)} d x+\frac{u_{R}}{R} .
$$

The value of $u_{R}$ should be found using the solution in the elastic region. Substituting (27) into (16) and using (29) and (30) give

$$
\frac{\partial\left(u_{\theta} / r\right)}{\partial \rho}=-\rho \frac{k_{0}}{G_{0}}
$$

The solution of this equation satisfying the boundary condition (2) is

$$
\frac{u_{\theta}}{r}=\frac{k_{0}}{2 G_{0}}\left(s^{2}-\rho^{2}\right)+\omega .
$$

It follows from (29) that $\rho=1$ if $r=R$. Therefore, the value of $u_{R}$ is determined from (36) as

$$
\frac{u_{R}}{R}=\frac{k_{0}}{2 G_{0}}\left(s^{2}-1\right)+\omega .
$$

Substituting (37) into (34) gives

$$
\frac{u_{\theta}}{r}=-\frac{1}{2} \int_{0}^{\varepsilon_{\mathrm{eq}}^{p}} \frac{\Phi(x) \Lambda^{\prime}(x)}{\Lambda(x)} d x+\frac{k_{0}}{2 G_{0}}\left(s^{2}-1\right)+\omega .
$$

Let $\varepsilon_{s}$ be the value of $\varepsilon_{\mathrm{eq}}^{p}$ at $r=r_{0}$. Then, combining (38) and the boundary condition (1) results in

$$
\int_{0}^{\varepsilon_{s}} \frac{\Phi\left(\varepsilon_{\mathrm{eq}}^{p}\right) \Lambda^{\prime}\left(\varepsilon_{\mathrm{eq}}^{p}\right)}{\Lambda\left(\varepsilon_{\mathrm{eq}}^{p}\right)} d \varepsilon_{\mathrm{eq}}^{p}=\frac{k_{0}}{G_{0}}\left(s^{2}-1\right)+2 \omega .
$$

It follows from (29) that $\rho=R / r_{0}$ at $r=r_{0}$, and, therefore, $\rho=s R_{0} / r_{0}$ at $r=r_{0}$. Then, (31) shows that $s^{2} R_{0}^{2} / r_{0}^{2}=\Lambda\left(\varepsilon_{s}\right)$. Using this equation to eliminate $s$ in (39) yields

$$
\int_{0}^{\varepsilon_{s}} \frac{\Phi\left(\varepsilon_{\mathrm{eq}}^{p}\right) \Lambda^{\prime}\left(\varepsilon_{\mathrm{eq}}^{p}\right)}{\Lambda\left(\varepsilon_{\mathrm{eq}}^{p}\right)} d \varepsilon_{\mathrm{eq}}^{p}-\frac{k_{0}}{G_{0}}\left[\frac{r_{0}^{2}}{R_{0}^{2}} \Lambda\left(\varepsilon_{s}\right)-1\right]=2 \omega .
$$

It follows from (23) that the value of $D$ at $r=r_{0}$ is given by

$$
D_{s}=\Omega^{-1}\left(\varepsilon_{s}-\varepsilon_{0}\right)
$$

It has been assumed here that $\varepsilon_{s} \geq \varepsilon_{0}$.

\section{Fracture Initiation and Nonexistence of the Solution}

Equation (40) determines $\varepsilon_{s}$ as a function of $\omega$. It follows from this equation that

$$
2 \frac{d \omega}{d \varepsilon_{s}}=\left[\frac{\Phi\left(\varepsilon_{s}\right)}{\Lambda\left(\varepsilon_{s}\right)}-\frac{k_{0}}{G_{0}} \frac{r_{0}^{2}}{R_{0}^{2}}\right] \Lambda^{\prime}\left(\varepsilon_{s}\right) .
$$

The right hand side of this equation vanishes if

$$
\frac{\Phi\left(\varepsilon_{s}\right)}{\Lambda\left(\varepsilon_{s}\right)}=\frac{k_{0}}{G_{0}} \frac{r_{0}^{2}}{R_{0}^{2}}
$$

or

$$
\Lambda^{\prime}\left(\varepsilon_{s}\right)=0
$$

Using (31) and (32), the left hand side of (43) can be represented in the form

$$
\frac{\Phi\left(\varepsilon_{s}\right)}{\Lambda\left(\varepsilon_{s}\right)}=\frac{k_{0}}{G_{0}} \frac{\left(A+\sqrt{3} \varepsilon_{s} G_{0} / k_{0}\right)}{A\left[1-\Omega^{-1}\left(\varepsilon_{s}-\varepsilon_{0}\right)\right]},
$$

where $A=1+f\left(\varepsilon_{s}\right)$. Consider the coefficient of $k_{0} / G_{0}$ in (45). It follows from the definition for the function $\Omega(D)$ that $\Omega^{-1}\left(\varepsilon_{s}-\varepsilon_{0}\right)$ is equal to the value of $D$ at $r=r_{0}$. Therefore, $0<1-\Omega^{-1}\left(\varepsilon_{s}-\varepsilon_{0}\right)<1$ and the denominator of the coefficient of $k_{0} / G_{0}$ in (45) is smaller than $A$. On the other hand, it is evident that its numerator is larger than $A$. Therefore, the coefficient is larger than 1 . However, the coefficient of $k_{0} / G_{0}$ in (43) is smaller than 1 . Hence (43) has no solution. Let $\varepsilon_{m}$ be the solution to (44). The corresponding values of $\omega=\omega_{m}$ and $D_{s}=D_{m}$ are determined from (40) and (41) at $\varepsilon_{s}=\varepsilon_{m}$. Expanding the left hand side of (42) in a series in the vicinity of $\varepsilon_{s}=\varepsilon_{m}$ gives $d \omega / d \varepsilon_{s}=\beta\left(\varepsilon_{s}-\varepsilon_{m}\right)$ to leading order. Here $\beta$ is constant. Integrating this equation with the use of the condition $\varepsilon_{s}=\varepsilon_{m}$ at $\omega=\omega_{m}$ results in $2\left(\omega-\omega_{m}\right)=$ $\beta\left(\varepsilon_{s}-\varepsilon_{m}\right)$ to leading order. This solution can be rewritten in the form $\varepsilon_{s}=\varepsilon_{m}-\sqrt{2 \beta^{-1}\left(\omega-\omega_{m}\right)}$. It is evident from this expression that $\varepsilon_{s}$ cannot exceed the value of $\varepsilon_{m}$. Thus if (43) has a solution, then the solution given in the previous section does not exist for $\omega \geq \omega_{m}$. On the other hand, according to the fracture criterion (11), the initiation of fracture at the bimaterial interface occurs if $D_{s}=D_{c}$. The corresponding value of $\varepsilon_{s}=\varepsilon_{c}$ is determined from (41) as $\varepsilon_{c}=\Omega\left(D_{c}\right)+\varepsilon_{0}$. Hence the solution to the boundary value problem of the previous section, including the fracture criterion, exists if and only if

$$
\varepsilon_{c} \leq \varepsilon_{m} \quad\left(\text { or } D_{c} \leq D_{m}\right)
$$

In particular, the solution predicts the initiation of fracture at the bimaterial interface at $\varepsilon_{s}=\varepsilon_{c}$. It will be shown in the next section that the inequality (46) is not satisfied for some specific damage evolution laws and constitutive parameters used in applications.

\section{Illustrative Example}

In the case under consideration the damage evolution model $[3,4]$ is obtained if $F_{1}(D)=\alpha$ where $\alpha$ is constant. Therefore, evaluating the integral in (24) gives

$$
\Omega(D)=\frac{D}{\alpha} \text {. }
$$

Then, it follows from (23) that

$$
\Omega^{-1}\left(\varepsilon_{\mathrm{eq}}^{p}-\varepsilon_{0}\right)=\alpha\left(\varepsilon_{\mathrm{eq}}^{p}-\varepsilon_{0}\right) .
$$


The equation of the strain hardening curve for the steel tested in [13], is

$$
f\left(\varepsilon_{\mathrm{eq}}^{p}\right)=2.78\left(\varepsilon_{\mathrm{eq}}^{p}\right)^{0.32} .
$$

Using experimental data provided in [13] it is possible to find that

$$
D_{c}=0.24, \quad \varepsilon_{0}=0.023, \quad \alpha=0.6 .
$$

Substituting (48)-(50) into (31) gives $\Lambda\left(\varepsilon_{\mathrm{eq}}^{p}\right)=[1+$ $\left.2.78\left(\varepsilon_{\mathrm{eq}}^{p}\right)^{0.32}\right]\left[1-0.6\left(\varepsilon_{\mathrm{eq}}^{p}-0.023\right)\right]$. Hence, $(44)$ becomes $0.89 \varepsilon_{m}^{-0.68}\left[1-0.6\left(\varepsilon_{m}-0.023\right)\right]-0.6\left(1+2.78 \varepsilon_{m}^{0.32}\right)=0$. Its solution is $\varepsilon_{m}=0.29$. Substituting this value of $\varepsilon_{\mathrm{eq}}^{p}=\varepsilon_{m}$ into (41) with the use of (48) gives $D_{m}=0.16$. This means that the solution to the boundary value problem without using the fracture criterion breaks down when $D_{s}=0.16$. On the other hand, the fracture criterion (11) is not satisfied as follows from (50). Therefore, it is necessary to conclude that the damage evolution model adopted is not compatible with the constitutive parameters recommended in [13].

\section{Conclusions}

An exact analytic solution for an elastic-plastic model coupled with a damage evolution equation has been found. Special attention has been devoted to the qualitative behavior of the solution in the vicinity of the bimaterial interface. Even though the boundary value problem solved is not feasible for practical applications, the main result obtained is useful for assessing damage evolution models by mathematical methods. In particular, it has been shown that the boundary value problem may have no solution because the equation relating the equivalent plastic strain at the bimaterial interface and one of the boundary conditions has no solution. This conclusion is not valid if the initiation of fracture occurs at a lower value of this strain. Therefore, having specific damage evolution equation and constitutive parameters, it is possible to verify that the model is compatible with conditions at bimaterial interfaces where shear straining prevails. As an example, the constitutive equations proposed in [13] have been used, and it has been demonstrated that the aforementioned equation has no solution. This means that some boundary value problems may have no solution for this model.

Even though (9) has been considered explicitly, it is evident that (10) can be treated in a similar manner. It is just necessary to replace (23) with

$$
\begin{aligned}
& \Omega(D)=E_{\mathrm{eq}}^{p}-E_{0} \text { if } \varepsilon_{\mathrm{eq}}^{p} \geq \varepsilon_{0}, \\
& D=0 \quad \text { if } \varepsilon_{\mathrm{eq}}^{p}<\varepsilon_{0},
\end{aligned}
$$

where $E_{\mathrm{eq}}^{p}=m^{-1}\left(\varepsilon_{\mathrm{eq}}^{p}\right)^{m}, E_{0}=m^{-1} \varepsilon_{0}^{m}$, and $m=1+M^{-1}$.

\section{Conflict of Interests}

The authors declare that there is no conflict of interests regarding the publication of this paper.

\section{Acknowledgment}

The research described in this paper has been supported by the Grants RFBR-11-01-00987 and NSH-3842.2012.1.

\section{References}

[1] Y. J. Liu, Q. Sun, X. L. Fan, and T. Suo, "A stress-invariant based multi-parameters ductile progressive fracture model," Materials Science and Engineering A, vol. 576, pp. 337-346, 2013.

[2] L. A. B. Cunda and G. J. Creus, "Mechanical response of porous materials: the Gurson model," in Plasticity of Pressure-Sensitive Materials, H. Altenbach and A. Öchsner, Eds., pp. 349-376, Springer, Berlin, Germany, 2014.

[3] J. Lemaitre, "A continuous damage mechanics model for ductile fracture," Transactions of ASME Journal of Engineering Materials and Technology, vol. 107, no. 1, pp. 83-89, 1985.

[4] J. Lemaitre, "Formulation and identification of damage kinetic constitutive equations," in Continuum Damage Mechanics: Theory and Applications, D. Krajcinovic and J. Lemaitre, Eds., pp. 37-89, Springer, Wien, Austria, 1987.

[5] S. Chandrakanth and P. C. Pandey, "A new ductile damage evolution model," International Journal of Fracture, vol. 60, no. 4, pp. R73-R76, 1993.

[6] P. Hartley, F. R. Hall, J. M. Chiou, and I. Pillinger, "Elasticplastic finite-element modelling of metal forming with damage evolution," in Advanced Methods in Materials Processing Defects, M. Predeleanu and P. Gilormini, Eds., pp. 135-142, Elsevier, Amsterdam, The Netherlands, 1997.

[7] S. Murakami, Continuum Damage Mechanics, Springer, Dordrecht, The Netherlands, 2012.

[8] S. Alexandrov and O. Richmond, "Singular plastic flow fields near surfaces of maximum friction stress," International Journal of Non-Linear Mechanics, vol. 36, no. 1, pp. 1-11, 2001.

[9] A. G. Atkins, "Fracture in forming," Journal of Materials Processing Technology, vol. 56, no. 1-4, pp. 609-618, 1996.

[10] I. F. Collins, "Boundary value problems in plane strain plasticity," in Mechanics of Solids, H. G. Hopkins and M. J. Sewell, Eds., pp. 135-184, Pergamon Press, Oxford, UK, 1982.

[11] S. Alexandrov and O. Richmond, "Couette flows of rigid/plastic solids: analytical examples of the interaction of constitutive and frictional laws," International Journal of Mechanical Sciences, vol. 43, no. 3, pp. 653-665, 2001.

[12] S. Alexandrov and G. Mishuris, "Viscoplasticity with a saturation stress: distinguishing features of the model," Archive of Applied Mechanics, vol. 77, no. 1, pp. 35-47, 2007.

[13] J. Lemaitre, "Coupled elasto-plasticity and damage constitutive equations," Computer Methods in Applied Mechanics and Engineering, vol. 51, no. 1-3, pp. 31-49, 1985. 


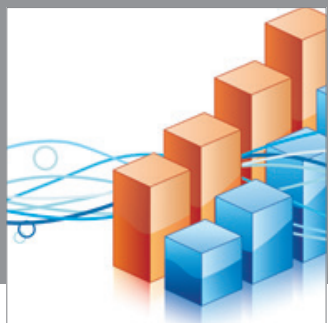

Advances in

Operations Research

mansans

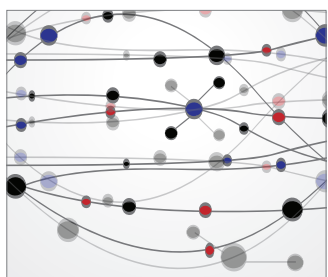

The Scientific World Journal
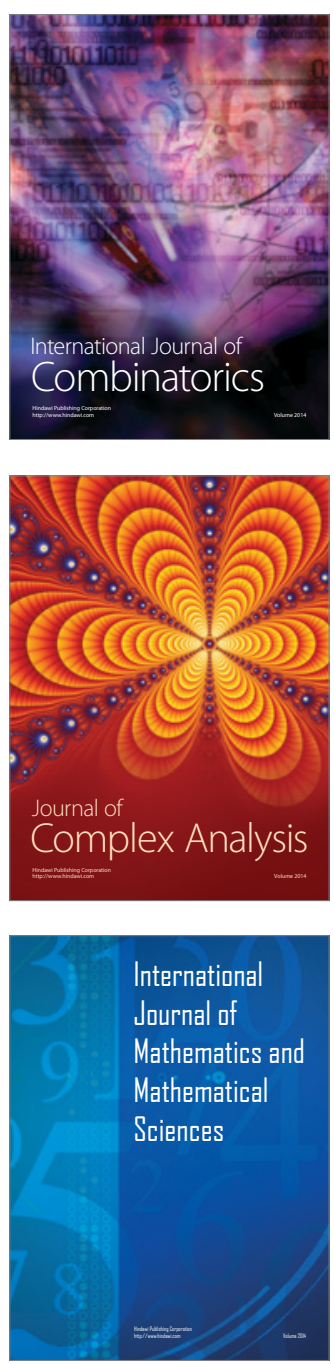
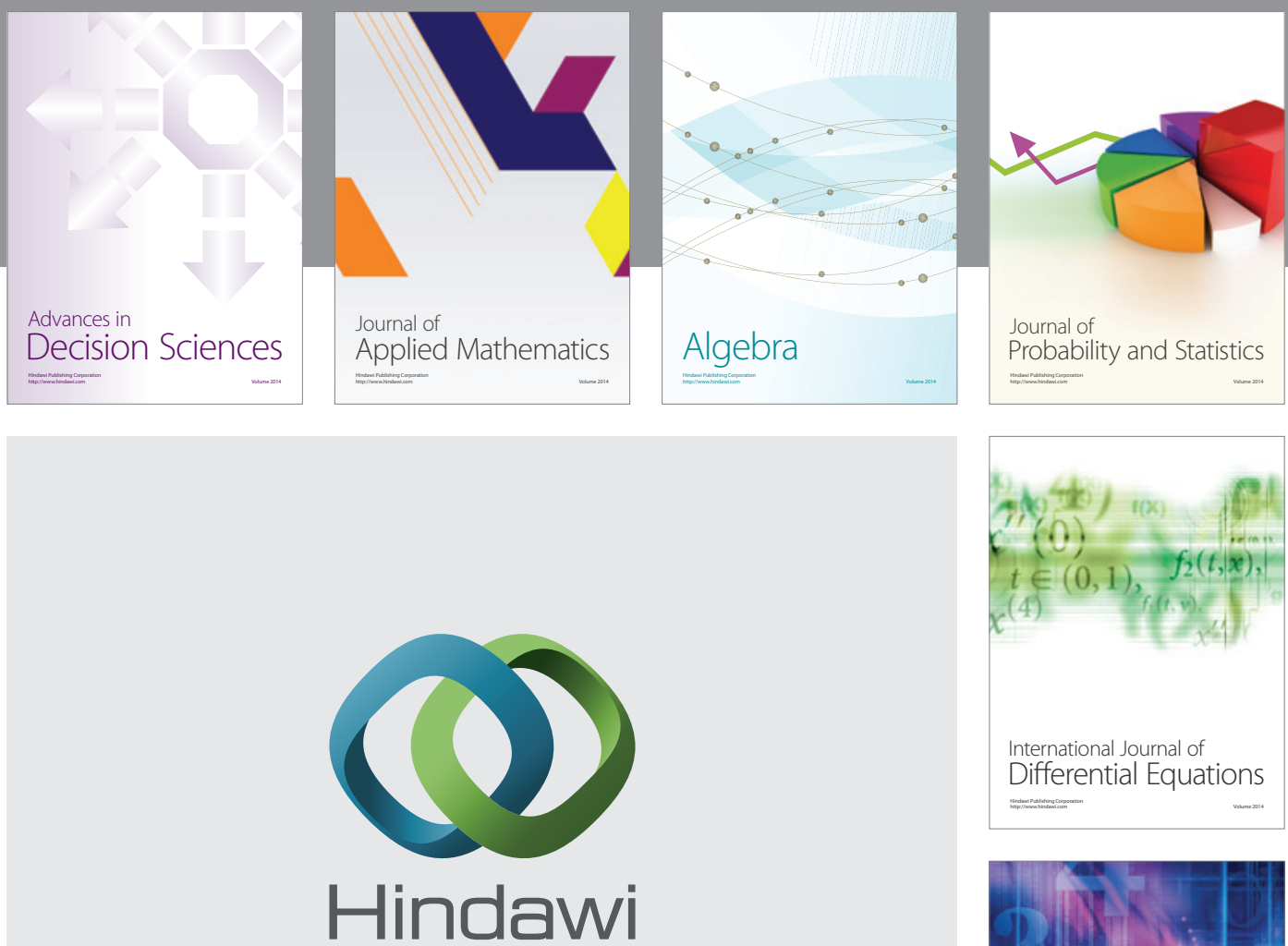

Submit your manuscripts at http://www.hindawi.com
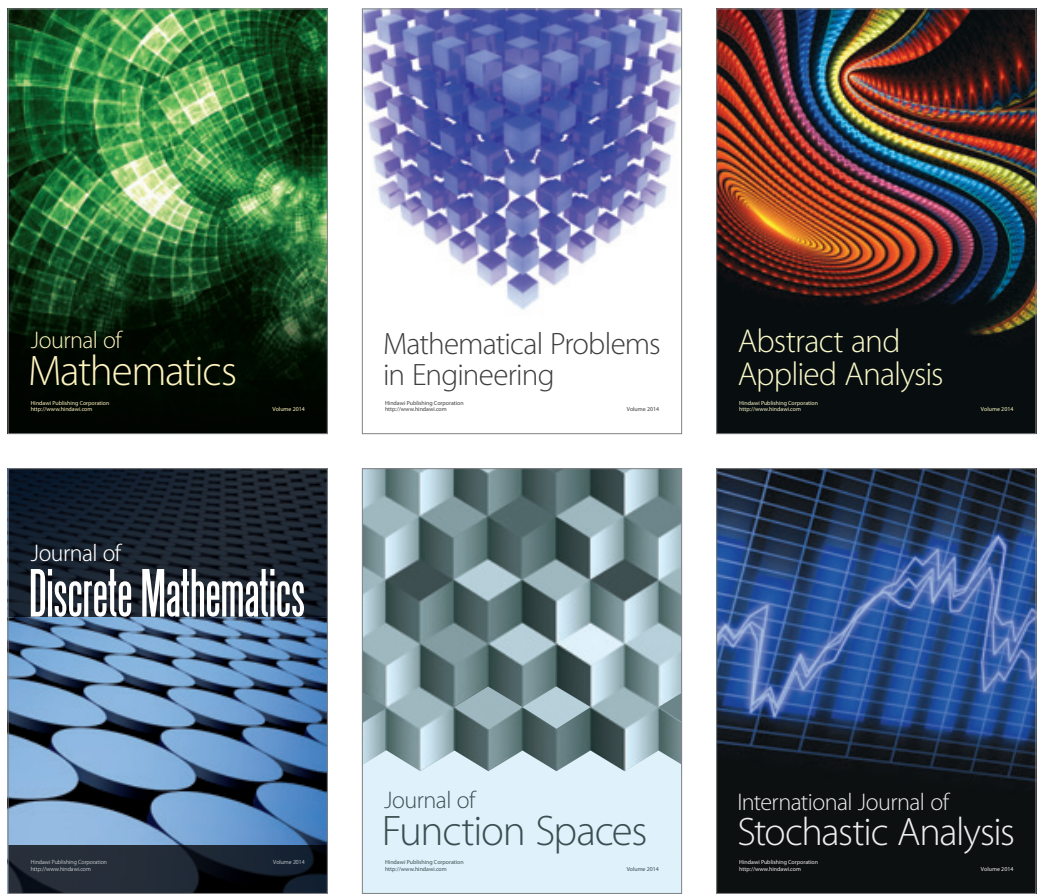

Journal of

Function Spaces

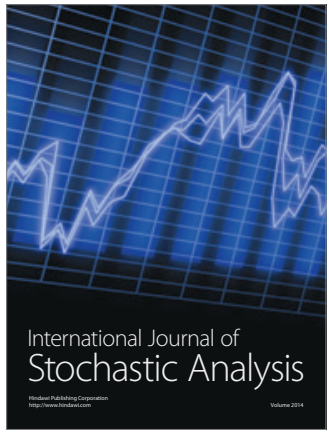

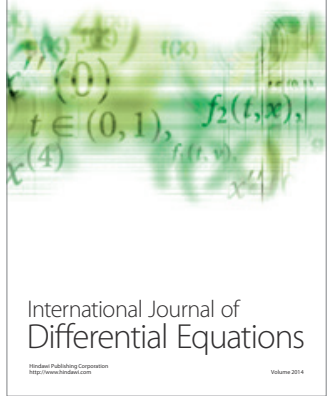
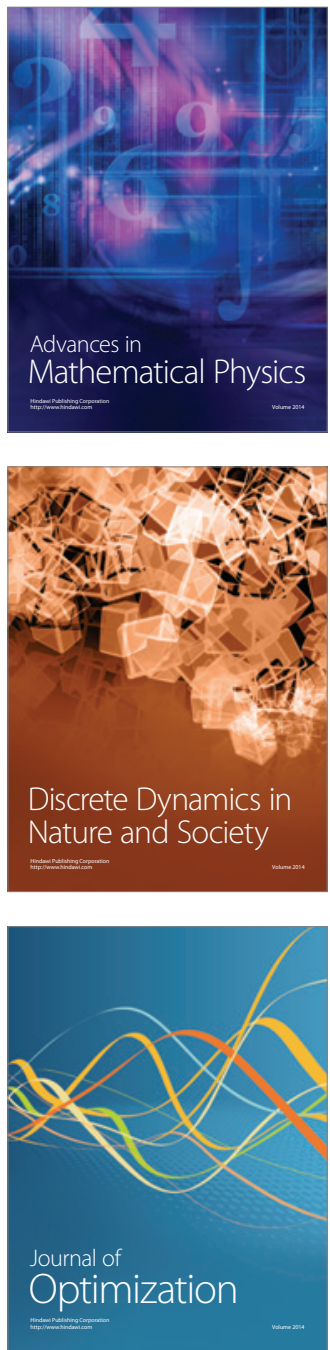